\title{
Iteration method for calculating the characteristic admittance matrix of multi-conductor transmission lines
}

\author{
Yixing Gu${ }^{1}$, Zhongyuan Zhou ${ }^{1 a)}$ and Mingjie Sheng ${ }^{1}$
}

Abstract It is necessary to solve the square root of the $\boldsymbol{L C}$ matrix when calculating the characteristic impedance matrix of multi-conductor transmission lines. This letter presents an algorithm based on the simple iteration method. There are no strict requirements for selecting initial values, which means that any initial value can converge to the correct solution. Based on this algorithm, a program is developed, and the solution is in agreement with other algorithm results.

key words: Algorithm, Iteration, Characteristic admittance matrix, Square root

Classification: Electromagnetic theory

\section{Introduction}

In the field of electrical engineering and electronic engineering, the multi-conductor transmission line is a very important electrical structure $[1,2,3]$. High-voltage transmission and distribution lines, power cables, communication cables, computer networks, and various printed circuit boards (PCB) are examples of the physical realization of multiconductor transmission lines. For a printed circuit board (PCB), it consists of a flat insulating board with a rectangular cross-section of conductors (ridges) laid on the board for the interconnection of digital and analog components $[4,5,6,7]$. Lossy transmission lines depict the transmissionrelated phenomena such as signal delay, crosstalk, and reflections at terminations and discontinuities with adequate accuracy, which is obtained at the price of complicated calculations [8]. It is simpler to model with lossless transmission lines. The analysis of the upper bound crosstalk and reflection generated by such a simplified signal transmission is acceptable in the design. The only caution is the signal lag, which is underestimated $[9,10,11]$.

For multi-conductor transmission lines, their distribution parameters cannot be ignored; crosstalk is probably the single most important factor affecting the high-speed transmission of data. It is an undesirable amount of energy produced by the coupling of one signal to another. [12, 13, 14, 15].

The capacitance and inductance matrices per line length, $C$

\footnotetext{
${ }^{1}$ Research Center for Electromagnetic Environmental Effects, Southeast University Nanjing, China

a)zyzhou@seu.edu.cn
}

DOI: $10.1587 /$ elex.18.20210343

Received August 17, 2021

Accepted September 13, 2021

Publicized September 21, 2021 and $\boldsymbol{L}$, are unique bridges between the physical and electrical characteristics of a lossless line system [14, 15, 16]. These matrices connect the voltages and currents of signals transmitted by the system along the transmission lines $[17,18]$. The $\boldsymbol{V}=\left[V_{1}, V_{2}, V_{3} \ldots, V_{n}\right]$ and $\boldsymbol{I}=\left[I_{1}, I_{2}, I_{3} \ldots, I_{n}\right]$ are connected by Eqs.(1) and (2).

$$
\begin{aligned}
& \frac{\partial}{\partial z} \boldsymbol{V}=-\boldsymbol{L} \frac{\partial}{\partial t} \boldsymbol{I} \\
& \frac{\partial}{\partial z} \boldsymbol{I}=-\boldsymbol{C} \frac{\partial}{\partial t} \boldsymbol{V}
\end{aligned}
$$

The characteristic admittance matrix is given by [8]:

$$
\boldsymbol{Y}_{0}=\boldsymbol{L}^{-1}(\boldsymbol{L} \boldsymbol{C})^{\frac{1}{2}}
$$

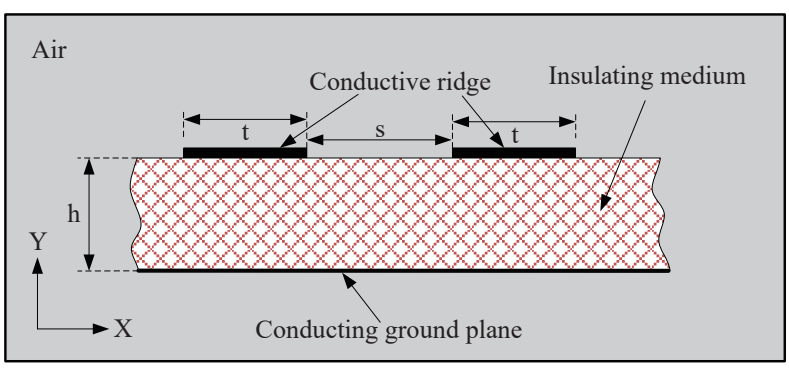

Fig. 1. Cross section of a tri-conductor transmission lines system.

The characteristic admittance matrix in the multiconductor transmission lines system is a significant quantity for designers, which directly affects the design of terminal matching network $[19,20]$.

Compared with the solution of the characteristic admittance in the two-conductor transmission lines, the solution of the characteristic admittance in the multi-conductor transmission lines is much more difficult [21, 22, 23, 24, 25]. Fig. 1 shows the cross-section of a tri-conductor transmission lines system, which is the model in this letter, extending in the $\mathrm{Z}$ direction. The two conductive ridges and the ground plane constitute a tri-conductor transmission lines system, and the insulating medium is between the conductive ridges and the ground plane.

In this letter, a simple iteration method is applied to calculate the characteristic admittance matrix. Part 2 mainly 
states the mathematical problems that need to be solved and the algorithms proposed by others. Part 3 introduces the algorithm adopted in this letter in detail. In part 4 , the characteristic admittance matrix of a tri-conductor transmission lines system is calculated with the algorithm in part 3 , and the result is in agreement with the result in part 2 .

\section{Existing algorithm}

In order to reduce or eliminate reflections, the terminal network of the line system should approximate or match the characteristic admittance matrix [26, 27, 28, 29, 30]. The computation of the matrix is based on Eq.(3), which involves the square root of the matrix product $\boldsymbol{L C}$. The calculation method of matrix $\boldsymbol{L}$ and matrix $\boldsymbol{C}$ respectively is given by [8]. Since the calculation method of matrix $\boldsymbol{L}$ and matrix $\boldsymbol{C}$ is not the emphasis of this letter, at the same time, in order to compare with the calculation results in the literature, the value of matrix $\boldsymbol{L}$ and matrix $\boldsymbol{C}$ are cited directly from [28] without explanation.

$$
\begin{gathered}
\boldsymbol{L}=\left[\begin{array}{ll}
436.57 & 127.94 \\
127.94 & 495.66
\end{array}\right] \mathrm{nH} / \mathrm{m} \\
\boldsymbol{C}=\left[\begin{array}{cc}
126.546 & -20.644 \\
-20.644 & 106.104
\end{array}\right] \mathrm{pF} / \mathrm{m} \\
\boldsymbol{L C}=\left[\begin{array}{cc}
52605 & 4562 \\
5958 & 49950
\end{array}\right](\mathrm{nH} / \mathrm{m}) \cdot(\mathrm{pF} / \mathrm{m})
\end{gathered}
$$

The most important calculation part of the formula Eq.(3) is the square root operation of the matrix $\boldsymbol{L C}$.

\subsection{Eigenvalue analysis method}

The classical solution method is to diagonalize the $\boldsymbol{L C}$ matrix through the matrix $\boldsymbol{P}$ [28].

$$
\begin{aligned}
& \boldsymbol{P}^{-1} \boldsymbol{L C P}=\Lambda^{2} \\
& \boldsymbol{L C}=(\boldsymbol{L C})^{\frac{1}{2}}(\boldsymbol{L C})^{\frac{1}{2}}=\boldsymbol{P} \Lambda \boldsymbol{P}^{-1} \boldsymbol{P} \Lambda \boldsymbol{P}^{-1}=\boldsymbol{P} \Lambda^{2} \boldsymbol{P}^{-1} \\
& (\boldsymbol{L C})^{\frac{1}{2}}=\boldsymbol{P} \Lambda \boldsymbol{P}^{-1}
\end{aligned}
$$

The right side of Eq.(7) is a diagonal matrix, $\Lambda^{2}$, matrix $\boldsymbol{P}$ is composed of eigenvectors, and then the square root of $\boldsymbol{L C}$ can be computed with eigenvalues and eigenvectors.

However, due to the inherent shortcomings of this method, if $\boldsymbol{L C}$ matrix is not a symmetric matrix, then it becomes challenging to solve the eigenvalues and eigenvectors of this matrix, especially when multiple eigenvalues exit.
2.2 multidimensional Newtonian iteration method

Karl [28] proposed a method that avoids the characteristic analysis and ambiguity associated with it and can be applied to other matrices.

Suppose that the square root of matrix $\boldsymbol{A}$ denoted $\boldsymbol{R}$ such that:

$$
\boldsymbol{R} \boldsymbol{R}=\boldsymbol{A}
$$

Where,

$$
\begin{aligned}
& \boldsymbol{R}=\left[\begin{array}{ccccc}
r_{11} & \cdots & r_{1 j} & \cdots & r_{1 n} \\
\vdots & \ddots & \vdots & \vdots & \vdots \\
r_{i 1} & \cdots & r_{i j} & \cdots & r_{i n} \\
\vdots & \vdots & \vdots & \ddots & \vdots \\
r_{n 1} & \cdots & r_{n j} & \cdots & r_{n n}
\end{array}\right]=\left[r_{i j}\right] \\
& \boldsymbol{A}=\left[\begin{array}{ccccc}
a_{11} & \cdots & a_{1 j} & \cdots & a_{1 n} \\
\vdots & \ddots & \vdots & \vdots & \vdots \\
a_{i 1} & \cdots & a_{i j} & \cdots & a_{i n} \\
\vdots & \vdots & \vdots & \ddots & \vdots \\
a_{n 1} & \cdots & a_{n j} & \cdots & a_{n n}
\end{array}\right]=\left[a_{i j}\right]
\end{aligned}
$$

As shown above, Eq.(10) is the problem of solving a nonlinear system of equations with $n^{2}$ unknowns, and can be rewritten in the following form as Eq.(13):

$$
\sum_{l=1}^{n} r_{i l} \cdot r_{l j}=a_{i j} ; 1 \leq i \leq n, 1 \leq j \leq n
$$

It is evident that the multidimensional Newton's method can be used to solve the roots of Eq.(13); therefore, Eq.(13) needs to be converted to something like Eq.(14):

$$
f_{i j}=\sum_{l=1}^{n} r_{i l} \cdot r_{l j}-a_{i j}=0
$$

The iterative format of multidimensional Newton's method can be constructed as Eq.(15):

$$
\boldsymbol{X}_{k+1}=\boldsymbol{X}_{k}-\boldsymbol{J}_{k}^{-1} \cdot \boldsymbol{G}_{k}
$$

Where, the subscript $k$ is the iteration counter, $\boldsymbol{X}$ is a $n^{2} \times 1$ dimensional matrix with $n^{2}$ unknowns as Eq.(16), $\boldsymbol{G}$ is a $n^{2} \times 1$ dimensional matrix as Eq.(17), $\boldsymbol{J}$ is a $n^{2} \times n^{2}$ dimensional matrix as Eq.(18).

$$
\begin{gathered}
\boldsymbol{X}=\left[\begin{array}{c}
r_{11} \\
r_{12} \\
\vdots \\
r_{i j} \\
\vdots \\
r_{n n}
\end{array}\right] \\
\boldsymbol{G}=\left[\begin{array}{c}
\sum_{l=1}^{n} r_{1 l} \cdot r_{l 1}-a_{11} \\
\sum_{l=1}^{n} r_{1 l} \cdot r_{l 2}-a_{12} \\
\vdots \\
\sum_{l=1}^{n} r_{n l} \cdot r_{l n}-a_{n n}
\end{array}\right]
\end{gathered}
$$




$$
\boldsymbol{J}=\left[\begin{array}{ccccc}
\frac{\partial f_{11}}{\partial r_{11}} & \frac{\partial f_{11}}{\partial r_{12}} & \cdots & \cdots & \frac{\partial f_{11}}{\partial r_{n n}} \\
\frac{\partial f_{12}}{\partial r_{11}} & \ddots & \frac{\partial f_{12}}{\partial r_{l m}} & \cdots & \vdots \\
\vdots & \vdots & \frac{\partial f_{i j}}{\partial r_{l m}} & \cdots & \frac{\partial f_{i j}}{\partial r_{n n}} \\
\vdots & \vdots & \vdots & \ddots & \vdots \\
\frac{\partial f_{n n}}{\partial r_{11}} & \cdots & \frac{\partial f_{n n}}{\partial r_{l m}} & \cdots & \frac{\partial f_{n n}}{\partial r_{n n}}
\end{array}\right]
$$

\section{The simple iteration method}

Although Newton's method has second-order accuracy, the convergence of Newton's method is determined by the selection of initial values, and not all initial values can ensure convergence. The initial values must be chosen close enough to the exact solution to ensure convergence [29]. To solve this problem, this letter proposes a method based on iteration to solve the square root operation of $\boldsymbol{L C}$ matrix.

In the following part of this section, a more efficient algorithm for directly calculating the square root of $\boldsymbol{L} \boldsymbol{C}$-matrix is proposed. Because it avoids the eigenvalue analysis and initial value selection, the algorithm is more general than the algorithm given in the literature, and it is suitable for an asymmetric square matrix. An example of calculating the square root of the asymmetric square matrix is shown in part 4.

Iterative method is a typical method in numerical calculation, which is not only used for solving equation, but also for solving eigenvalues of matrices. The basic idea of this method is a method of successive approximation, which first takes a rough approximation and then verifies the solution from the last iteration repeatedly with a recursive formula until the predetermined accuracy requirements are met. So this letter constructs an appropriate recursion formula.

Therefore, the problem can be thought of as requiring the solution of a system of equations containing $n^{2}$ equations as Eq.(13).

The key to the algorithm proposed in this letter is that $r_{i j}$ can be represented by terms that do not contain $r_{i j}$ (see Eq.(19) and Eq.(20)).

If $i=j$,

$$
r_{i j}=\left(a_{i j}-\left(\sum_{l=1}^{n}\left(r_{i l} \cdot r_{l j}\right)-r_{i j} \cdot r_{i j}\right)\right)^{\frac{1}{2}}
$$

If $i \neq j$,

$r_{i j}=\frac{1}{r_{i i}+r_{j j}}\left(a_{i j}-\left(\sum_{l=1}^{n} r_{i l} \cdot r_{l j}-r_{i i} \cdot r_{i j}-r_{j j} \cdot r_{i j}\right)\right)$

It can be expressed in matrix form $(i \neq j$ in Eq.(21)).

$$
\begin{aligned}
{\left[\begin{array}{c}
r_{11} \\
r_{12} \\
\vdots \\
r_{i j} \\
\vdots \\
r_{n n}
\end{array}\right] } & =\left[\begin{array}{c}
\left(a_{11}-\left(\sum_{l=1}^{n}\left(r_{1 l} \cdot r_{l 1}\right)-r_{11} \cdot r_{11}\right)\right)^{\frac{1}{2}} \\
\frac{1}{r_{11}+r_{22}}\left(a_{12}-\left(\sum_{l=1}^{n} r_{1 l} \cdot r_{l 2}-r_{11} \cdot r_{12}-r_{22} \cdot r_{12}\right)\right) \\
\vdots \\
\frac{1}{r_{i i}+r_{j j}}\left(a_{i j}-\left(\sum_{l=1}^{n} r_{i l} \cdot r_{l j}-r_{i i} \cdot r_{i j}-r_{j j} \cdot r_{i j}\right)\right) \\
\vdots \\
\left(a_{n n}-\left(\sum_{l=1}^{n}\left(r_{n l} \cdot r_{l n}\right)-r_{n n} \cdot r_{n n}\right)\right)^{\frac{1}{2}}
\end{array}\right] \\
& =\boldsymbol{F}\left(\left[\begin{array}{c}
r_{11} \\
r_{12} \\
\vdots \\
r_{i j} \\
\vdots \\
r_{n n}
\end{array}\right]\right)
\end{aligned}
$$

The equations above can be expressed as:

$$
\begin{gathered}
X=F(X) \\
F(X)-X=0
\end{gathered}
$$

Therefore, the following iteration format can be constructed:

$$
\boldsymbol{X}_{k+1}=\boldsymbol{F}\left(\boldsymbol{X}_{k}\right)
$$

If the solution precision $\epsilon$ is satisfied, which means \| $\boldsymbol{X}_{k+1}-\boldsymbol{X}_{k} \| \leq \epsilon$. As a result, $\boldsymbol{X}_{k+1}$ can be thought of as a solution to Eq.(22).

Attention needs to be paid here in Eq.(21) if $r_{i i} \cdot r_{i i}<$ 0 , even if the case of $r_{i i} \cdot r_{i i}<0$ happens in the iteration process, that is, $r_{i i}$ is an imaginary number, the algorithm will still be executed and converge to the correct solution.

\section{Solution of positive definite matrix in a multi- conductor transmission lines system}

The calculation results of the algorithm proposed by this letter are shown in Table I with a solution precision $\epsilon=$ $10^{-6}$, where the selected initial value is a zero matrix.

$$
\begin{array}{r}
\boldsymbol{L C}=\left[\begin{array}{cc}
52605 & 4562 \\
5958 & 49950
\end{array}\right](\mathrm{nH} / \mathrm{m}) \cdot(\mathrm{pF} / \mathrm{m}) \\
=\left[\begin{array}{cc}
52.6050 & 4.5624 \\
5.9579 & 49.9503
\end{array}\right] \times 10^{-18}(\mathrm{H} / \mathrm{m}) \cdot(\mathrm{F} / \mathrm{m})
\end{array}
$$

Since $1 \mathrm{H} / \mathrm{m} \times 1 \mathrm{~F} / \mathrm{m}=10^{9} \mathrm{nH} / \mathrm{m} \times 10^{12} \mathrm{pF} / \mathrm{m}=$ $10^{21}(\mathrm{nH} / \mathrm{m}) \cdot(\mathrm{pF} / \mathrm{m})$, the matrix product $\boldsymbol{L C}$ is multiplied by a coefficient of $10^{18}$ to compare with the results in the literature. 
The iteration progress of matrix $\boldsymbol{R}$ with other initial values are also given by Table II - Table V. For an arbitrary initial value (strictly speaking, any initial value that has been tried so far), the algorithm is convergent; any iteration that starts with any initial value that we set converges to the same solution, which is consistent with the results obtained by iterative solution of multidimensional Newton method (see Table VI) in [28]. It is clearly demonstrated that this method does not rely on suitable initial values as much as the method in [28].

Table I. The results calculated by proposed algorithm SQUARE ROOT OF A REAL POSITIVE DEFINITE MATRIX

\begin{tabular}{ccccc}
\hline \multicolumn{5}{c}{ INPUT ORIGINAL $2 \times 2$ MATRIX A } \\
$\mathrm{A}$ & $\mathrm{A}_{11}$ & $\mathrm{~A}_{12}$ & $\mathrm{~A}_{21}$ & $\mathrm{~A}_{22}$ \\
VALUE & 52.6050 & 4.5624 & 5.9579 & 49.9503 \\
\hline \multicolumn{5}{c}{ THE ITERATION PROGRESS } \\
\hline ITERATION & \multirow{5}{c}{ Elements of matrix R } \\
COUNT & $\mathrm{R}_{11}$ & $\mathrm{R}_{12}$ & $\mathrm{R}_{21}$ & $\mathrm{R}_{22}$ \\
\hline INITIAL & 0 & 0 & 0 & 0 \\
VALUE & & & & \\
\hline 1 & 7.25293 & 0.629042 & 0.821447 & 7.03090 \\
2 & 7.21722 & 0.320211 & 0.418153 & 7.05807 \\
3 & 7.24369 & 0.319010 & 0.416585 & 7.05814 \\
4 & 7.24376 & 0.319006 & 0.416581 & 7.05814 \\
5 & 7.24376 & 0.319006 & 0.416581 & 7.05814 \\
6 & 7.24376 & 0.319006 & 0.416581 & 7.05814
\end{tabular}

Table II. Iteration process under different initial values (A) SQUARE ROOT OF A REAL POSITIVE DEFINITE MATRIX

\begin{tabular}{|c|c|c|c|c|}
\hline \multicolumn{5}{|c|}{ INPUT ORIGINAL $2 \times 2$ MATRIX A } \\
\hline A & $\mathrm{A}_{11}$ & $\mathrm{~A}_{12}$ & $\mathrm{~A}_{21}$ & $\mathrm{~A}_{22}$ \\
\hline VALUE & 52.6050 & 4.5624 & 5.9579 & 49.9503 \\
\hline \multicolumn{5}{|c|}{ THE ITERATION PROGRESS } \\
\hline ITERATION & \multicolumn{4}{|c|}{ Elements of matrix R } \\
\hline COUNT & $\mathrm{R}_{11}$ & $\mathrm{R}_{12}$ & $\mathrm{R}_{21}$ & $\mathrm{R}_{22}$ \\
\hline $\begin{array}{l}\text { INITIAL } \\
\text { VALUE }\end{array}$ & 1 & 1 & 1 & 1 \\
\hline 1 & 7.183662 & 0.635108 & 0.829368 & 7.030189 \\
\hline 2 & 7.216527 & 0.320242 & 0.418195 & 7.058072 \\
\hline 3 & 7.243692 & 0.319010 & 0.416585 & 7.058145 \\
\hline 4 & 7.243763 & 0.319006 & 0.416581 & 7.058145 \\
\hline 5 & 7.243763 & 0.319006 & 0.416581 & 7.058145 \\
\hline 6 & 7.243763 & 0.319006 & 0.416581 & 7.058145 \\
\hline
\end{tabular}

Table III. Iteration process under different initial values (B) SQUARE ROOT OF A REAL POSITIVE DEFINITE MATRIX

\begin{tabular}{ccccc}
\hline \multicolumn{5}{c}{ INPUT ORIGINAL $2 \times 2$ MATRIX A } \\
$\mathrm{A}$ & $\mathrm{A}_{11}$ & $\mathrm{~A}_{12}$ & $\mathrm{~A}_{21}$ & $\mathrm{~A}_{22}$ \\
VALUE & 52.6050 & 4.5624 & 5.9579 & 49.9503 \\
\hline \multicolumn{5}{c}{ THE ITERATION PROGRESS } \\
\hline $\begin{array}{c}\text { ITERATION } \\
\text { COUNT }\end{array}$ & $\mathrm{R}_{11}$ & $\mathrm{R}_{12}$ & $\mathrm{R}_{21}$ & $\mathrm{R}_{22}$ \\
\hline INITIAL & 100 & 100 & 100 & 100 \\
VALUE & & & \\
\hline 1 & $99.737 \mathrm{i}$ & $-0.0457 \mathrm{i}$ & $-0.0597 \mathrm{i}$ & 7.067746 \\
2 & 7.253119 & 0.318584 & 0.416029 & 7.058170 \\
3 & 7.243788 & 0.319005 & 0.416579 & 7.058145 \\
4 & 7.243763 & 0.319006 & 0.416581 & 7.058145 \\
5 & 7.243763 & 0.319006 & 0.416581 & 7.058145 \\
6 & 7.243763 & 0.319006 & 0.416581 & 7.058145
\end{tabular}

Table IV. Iteration process under different initial values (C)

\begin{tabular}{ccccc}
\hline \multicolumn{5}{c}{ SQUARE ROOT OF A REAL POSITIVE DEFINITE MATRIX } \\
\hline \multicolumn{5}{c}{ INPUT ORIGINAL $2 \times 2$ MATRIX A } \\
$\mathrm{A}$ & $\mathrm{A}_{11}$ & $\mathrm{~A}_{12}$ & $\mathrm{~A}_{21}$ & $\mathrm{~A}_{22}$ \\
VALUE & 52.6050 & 4.5624 & 5.9579 & 49.9503 \\
\hline \multicolumn{5}{c}{ THE ITERATION PROGRESS } \\
\hline $\begin{array}{c}\text { ITERATION } \\
\text { COUNT }\end{array}$ & $\mathrm{R}_{11}$ & $\mathrm{R}_{12}$ & $\mathrm{R}_{21}$ & $\mathrm{R}_{22}$ \\
\hline INITIAL & 19 & 94 & 11 & 15 \\
VALUE & & & & \\
\hline 1 & $31.3272 \mathrm{i}$ & $-0.1456 \mathrm{i}$ & $-0.1902 \mathrm{i}$ & 7.069512 \\
2 & 7.254840 & 0.318507 & 0.415928 & 7.058174 \\
3 & 7.243792 & 0.319005 & 0.416579 & 7.058145 \\
4 & 7.243763 & 0.319006 & 0.416581 & 7.058145 \\
5 & 7.243763 & 0.319006 & 0.416581 & 7.058145 \\
6 & 7.243763 & 0.319006 & 0.416581 & 7.058145
\end{tabular}

Table V. Iteration process under different initial values (D)

\begin{tabular}{ccccc}
\multicolumn{5}{c}{ SQUARE ROOT OF A REAL POSITIVE DEFINITE MATRIX } \\
\hline \multicolumn{5}{c}{ INPUT ORIGINAL $2 \times 3$ MATRIX A } \\
A & $\mathrm{A}_{11}$ & $\mathrm{~A}_{12}$ & $\mathrm{~A}_{21}$ & $\mathrm{~A}_{22}$ \\
VALUE & 52.6050 & 4.5624 & 5.9579 & 49.9503 \\
\hline \multicolumn{5}{c}{ THE ITERATION PROGRESS } \\
\hline $\begin{array}{c}\text { ITERATION } \\
\text { COUNT }\end{array}$ & $\mathrm{R}_{11}$ & $\mathrm{R}_{12}$ & $\mathrm{R}_{21}$ & $\mathrm{R}_{22}$ \\
\hline INITIAL & 20 & 21 & 4 & 30 \\
VALUE & & & & \\
\hline 1 & $5.60312 \mathrm{i}$ & $-0.8143 \mathrm{i}$ & $-1.0633 \mathrm{i}$ & 7.069512 \\
2 & 7.312374 & 0.315936 & 0.412571 & 7.058325 \\
3 & 7.243939 & 0.318998 & 0.416570 & 7.058145 \\
4 & 7.243764 & 0.319006 & 0.416581 & 7.058145 \\
5 & 7.243764 & 0.319006 & 0.416581 & 7.058145 \\
6 & 7.243764 & 0.319006 & 0.416581 & 7.058145
\end{tabular}

Table VI. The results in the literature SQUARE ROOT OF A REAL POSITIVE DEFINITE MATRIX

\begin{tabular}{ccccc}
\hline \multicolumn{5}{c}{ INPUT ORIGINAL $2 \times 2$ MATRIX A } \\
$\mathrm{A}$ & $\mathrm{A}_{11}$ & $\mathrm{~A}_{12}$ & $\mathrm{~A}_{21}$ & $\mathrm{~A}_{22}$ \\
VALUE & 52.6050 & 4.5624 & 5.9579 & 49.9503 \\
\hline \multicolumn{5}{c}{ THE ITERATION PROGRESS } \\
\hline ITERATION & $\mathrm{R}_{11}$ & $\mathrm{R}_{12}$ & $\mathrm{R}_{21}$ & $\mathrm{R}_{22}$ \\
COUNT & 7.25293 & 0.31859 & 0.41604 & 7.06755 \\
\hline 1 & 7.24377 & 0.31901 & 0.41658 & 7.05815 \\
2 & 7.24376 & 0.31901 & 0.41658 & 7.05814 \\
3 & 7.24376 & 0.31901 & 0.41658 & 7.05814 \\
4 & 7.24376 & 0.31901 & 0.41658 & 7.05814 \\
5 & 7.24376 & 0.31901 & 0.41658 & 7.05814 \\
6 & & & &
\end{tabular}

According to formula Eq.(3) and the algorithm in part 3,

$$
\boldsymbol{Y}_{0}=\left[\begin{array}{cc}
17.6838 & -3.7241 \\
-3.7241 & 15.2012
\end{array}\right] m S
$$

From the above results, the algorithm proposed in this paper converges to the final solution after four iterations, while the method in the literature only takes three iterations; from this perspective, the method in [28] takes advantage over the algorithm in this letter; however, it is clearly shown that the method proposed in this letter is superior to the method in the literature in terms of the selection of initial values. Compared to an algorithm where any initial value 
converges, the algorithm in [28], even though needing fewer iterations, is actually more complicated because the initial value has to be estimated. In terms of calculation time, both methods take less than 1 second in the context of computer programming calculations.

\section{Conclusion}

The algorithm presented in this letter can be applied to any square matrix. it is simple and practical, avoiding the shortcomings of the eigenanalysis and the multidimensional Newton iteration. If the matrix is symmetric, it is equivalent to reducing the number of unknowns to be solved, simplifying the algorithm. In some multi-conductor transmission lines systems, the $\boldsymbol{L}$ and $\boldsymbol{C}$ are positive definite matrices, so the $\boldsymbol{L C}$ is also a positive definite matrix, in this case the algorithm can be simplified.

\section{References}

[1] Y. Huangfu, et al.:"FDTD Formulation Based on HighOrder Surface Impedance Boundary Conditions for Frequency-Dependent Lossy Multi-Conductor Transmission Lines," IEEE Trans. Magn. 56 (2020) 7502304 (DOI: 10.1109/TMAG.2019.2950603) .

[2] D. Bormann and H. Tavakoli:"Reluctance Network Treatment of Skin and Proximity Effects in Multi-Conductor Transmission Lines," IEEE Trans. Magn. 48 (2012)735-738 (DOI: 10.1109/TMAG.2011.2175711) .

[3] S. M. H. Hosseini and P. R. Baravati: "New high frequency multi-conductor transmission line detailed model of transformer winding for PD study," IEEE T Dielect El In. 24 (2017) 316-323 (DOI: 10.1109/TDEI.2016.005694).

[4] Q. Zhang,et al.: "Application of an Improved MultiConductor Transmission Line Model in Power Transformer," IEEE Trans. Magn. 49 (2013) 2029-2032 (DOI: 10.1109/TMAG.2013.2245874) .

[5] J. Zhang ,et al:: "Analysis of Inter-Turn Insulation of High Voltage Electrical Machine by Using Multi-Conductor Transmission Line Model," IEEE Trans. Magn. 49 (2013) 1905-1908 (DOI: 10.1109/TMAG.2013.2245873).

[6] Y. Huangfu, et al.: "Frequency-Dependent Multi-Conductor Transmission Line Model for Shielded Power Cables Considering Geometrical Dissymmetry,” IEEE Trans. Magn. 54 (2018) 6300104 (DOI: 10.1109/TMAG.2017.2751958).

[7] M. Tsuda ,et al.:"Influence of current distribution on conductor performance in coaxial multi-layer HTS conductor," IEEE Trans. Appl Supercon. 12 (2002) 1643-1646 (DOI: 10.1109/TASC.2002.1018721).

[8] Clayton R. Paul: Analysis of Multi-conductor Transmission Lines, John Wiley\&Sons,ISBN 978-0-470-13154-1.

[9] Fung-Yuel Chang: "Transient Analysis of Lossless Coupled Transmission Lines in a Nonhomogeneous Dielectric Medium," IEEE Trans. Microw Theory Techn. 18 (1970) 616-626 (DOI: 10.1109/TMTT.1970.1127297).

[10] Tiebing Lu,et al.:"Transient analysis of aerial multiconductor transmission lines with branch," IEEE Trans. Magn. 27 (2001) 3298-3302 (DOI: 10.1109/20.952599).

[11] Qingxi Yang, et al.: "Fast transient analysis method for lossy nonuniform transmission line with nonlinear terminations," IEICE Electron. Express. 12 (2015) 20150362 (DOI: 10.1587/elex.12.20150362).

[12] Qingxi Yang, et al.: "Equivalent circuits of transmission lines sag above lossy ground excited by external electromag- netic fields," IEICE Electron. Express. 16 (2019) 20190102 (DOI:10.1587/elex.16.20190102).

[13] H. B. Lu, et al.: "Measurement of LC dielectric constant at lower terahertz region based on metamaterial absorber," IEICE Electron. Express. 14 (2017) 20170469 (DOI: 10.1587/elex.14.20170469).

[14] D. M. Pozar: Microwave engineering (Publishing House of Electronics Industry, Beijing, 2011) 3nd ed. 48.

[15] J. J. Yin: Microwave technology and antenna (Publishing House of Electronics Industry, Beijing, 2012) 2nd ed. 175.

[16] G. Antonini and L. De Camillis: "Time-Domain Green's Function-Based Sensitivity Analysis of Multiconductor Transmission Lines With Nonlinear Terminations," IEEE Microw. Wirel Co. 19 (2009) 428-430 (DOI: 10.1109/LMWC.2009.2022120).

[17] Y. Huangfu, et al.:"Modeling and Insulation Performance Analysis of Composite Transmission Line Tower Under Lightning Overvoltage," IEEE Trans. Magn. 51 (2015) 8001404 (DOI: 10.1109/TMAG.2014.2356859) .

[18] D. Bormann and H. Tavakoli: "Reluctance Network Method for Calculating the Series Impedance Matrix of MultiConductor Transmission Lines," IEEE Trans.Magn.49 (2013) 5270-5279 (DOI: 10.1109/TMAG.2013.2261999).

[19] S. Kim, et al.:"Compact Two-Way and Four-Way Power Dividers Using Multi-Conductor Coupled Lines," IEEE Microw. Wirel Co. 21 (2011) 130-132 (DOI: 10.1109/LMWC.2010.2100078) .

[20] Y. Zhang, et al:: "Analysis of Nonuniform Transmission Lines With a Perturbation Technique in Time Domain," IEEE Trans. Electromagn. Compat. 62 (2020) 542-548 (DOI: 10.1109/TEMC.2019.2906251) .

[21] H. M. J. De Silva,et al.:"Robust Passivity Enforcement Scheme for Time-Domain Simulation of MultiConductor Transmission Lines and Cables,'IEEE Trans. Power Deliv.25 (2010) 930-938 (DOI: 10.1109/TPWRD.2009.2035916).

[22] T. R. Arabi,et al.: "Electric field integral equation formulation for a dynamic analysis of nonuniform microstrip multiconductor transmission lines," IEEE Trans. Microw. Theory Techn. 40 (1992) 1857-1869 (DOI: 10.1109/22.159622).

[23] G. M. Casolino and A. Losi:"On Eigenvalues of the Three-Phase Nodal Admittance Matrix," 2019 AEIT International Annual Conference (AEIT) (2019) (DOI:10.23919/AEIT.2019.8893321).

[24] A. M. Kettner and M. Paolone,"On the Properties of the Compound Nodal Admittance Matrix of Polyphase Power Systems," IEEE Trans.Power Syst. 34 (2019) 444-453 (DOI: 10.1109/TPWRS.2018.2863671).

[25] L. Gong,et al:: "Admittance matrix of a symmetrical triple-layer piezoelectric cantilever," Proceedings of the 2014 Symposium on Piezoelectricity, Acoustic Waves, and Device Applications. (2014) 322-326 (DOI: 10.1109/SPAWDA.2014.6998591).

[26] A. Semlyen and B. Gustavsen: "Phase-Domain Transmission-Line Modeling With Enforcement of Symmetry Via The Propagated Characteristic Admittance Matrix," IEEE Trans. Power Deliv. 27 (2012) 626-631 (DOI: 10.1109/TPWRD.2011.2180405).

[27] Y. You, et al.: "New matrix algorithm for calculating diagonally matched impedance of packaging interconnecting lines," IEEE Trans. Microw. Theory Techn. 47 (1999)798801 (DOI: $10.1109 / 22.769353)$.

[28] K. Reiss and O. A. Palusinski: "Procedure for direct calculation of characteristic admittance matrix of coupled transmission lines," IEEE Transactions on Microw. Theory Techn. 44 (1996) 152-154 (DOI: 10.1109/22.481397). 
[29] Sun Zhizhong,et al:: A PRACTICAL NUMERICAL ANALYSIS Southeast University Press, ISBN 978-7-5641-2577-6.

[30] L. A. Hayden and V. K. Tripathi, "Characterization and modeling of multiple line interconnections from time domain measurements," IEEE Trans. Microw. Theory Techn. 42 (1994) 1737-1743 (DOI: 10.1109/22.310582). 